\title{
Face Tracking in the Compressed Domain
}

\author{
Pedro Miguel Fonseca and Jan Nesvadba \\ Philips Research, 5656AA Eindhoven, The Netherlands \\ Received 30 August 2004; Revised 23 March 2005; Accepted 4 May 2005
}

\begin{abstract}
A compressed domain generic object tracking algorithm offers, in combination with a face detection algorithm, a low-computational-cost solution to the problem of detecting and locating faces in frames of compressed video sequences (such as MPEG-1 or MPEG-2). Objects such as faces can thus be tracked through a compressed video stream using motion information provided by existing forward and backward motion vectors. The described solution requires only low computational resources on CE devices and offers at one and the same time sufficiently good location rates.
\end{abstract}

Copyright (C) 2006 P. M. Fonseca and J. Nesvadba. This is an open access article distributed under the Creative Commons Attribution License, which permits unrestricted use, distribution, and reproduction in any medium, provided the original work is properly cited.

\section{INTRODUCTION}

The problem of tracking objects over time is a complex one in computer vision and has been an important topic of research over the last few years. Such importance comes from the fact that object tracking enables important applications in areas such as security and surveillance (e.g., tracking people in restricted areas using security cameras), content management (e.g., in video abstraction to automatically annotate video content), content improvement (e.g., helping stabilize images in handheld mobile videophones by tracking the location of faces), human-machine interface (e.g., to automatically recognize hand gestures to automatically execute commands), interactive gaming, and so forth. Requirement constraints such as reliability and computational complexity characterize the boundary conditions for a successful and target-platform-suited solution.

The detection and spatial localization of objects, in particular faces, has been broadly investigated $[1,2]$. While tracking identified objects throughout uncompressed video sequences, the objects' spatial properties may be used (e.g., colour, shape, texture, etc.) since it can be expected that they will vary a little from frame to frame. The information is thus represented in a way suited to easily track the objects. However, in compressed video sequences (such as MPEG-1 or MPEG-2), available information may not express directly the objects' spatial properties and thus, renders the tracking procedure more difficult. In addition, the type of information that is available actually varies from frame to framefor example MPEG-1 or MPEG-2 video sequences are typically comprised of I-, P-, and B-frames, each with its own set of parameters. In this paper we describe an object tracking solution that uses only compressed parameters available in MPEG-1 or MPEG-2 video sequences while performing only the minimal decoding necessary to retrieve them from the compressed video streams.

Few algorithms exist that are able to perform object tracking in the compressed domain. In $[3,4]$ the proposed object tracking algorithms use forward motion vectors to track objects between a reference (I- or P-) frame and a Por B-frame (where this kind of motion vectors are available). In order to track an object, it is first defined as a set of macroblocks in the reference frame. However, the forward motion vectors are available only in the P- or B-frame where the object is being tracked to. So, the object's displacement from the reference frame to the P- or B-frame is determined by computing the mode of the forward motion vectors of the macroblocks at the same positions (in the latter frame) as those that define the object (in the reference frame). Although simple and not at all computationally complex, this solution is only reliable whenever motion is not strong or when motion is uniform over the entire frame.

In [5], an object is represented by a set of macroblocks in the reference frame. Each forward motion vector in the current (P- or B-) frame is "projected" in the reference frame and it is determined if it does or does not "hit" any macroblock that comprises the object. Afterwards, a $k$-means clustering algorithm is used to determine clusters of motion vectors that may represent possible object displacements. Although robust, this technique is considerably complex and computationally expensive. 
In the algorithms proposed in [6-9], objects can only be tracked to P- and I-frames, but not to B-frames.

The object tracking algorithm proposed in this paper performs the tracking of objects that are detected and located throughout a group of pictures (GOP) by means of available compressed domain data. We will describe a tracking solution based solely on the use of forward and backward motion vectors. A smart weighting and averaging strategy of relevant motion vectors has been proven to be sufficiently reliable for the purpose at hand. Additionally, the solution's computational complexity is extremely low enabling the implementation on platforms with very strict computational capacities such as mobile and stationary consumer devices. Although the proposed algorithm allows the tracking of generic objects, it will be described in the context of a face tracking system where the objects being tracked correspond to faces. Faces are detected in I-frames with a compressed domain face detector which is also briefly described.

The organization of this paper is as follows: the next section describes the face tracking algorithm; Section 3 evaluates the algorithm's computational complexity and the algorithm's performance; and the paper concludes with Section 4.

\section{THE FACE TRACKING ALGORITHM}

The face tracking algorithm proposed in this paper aims to determine the presence and location of faces in all frames of compressed video sequences where a specific type of compressed domain data is available. Naturally, the type of information available differs according to the type of frames where faces are being located: while in I-frames only DCT information is available, in $\mathrm{P}$ - and $\mathrm{B}$-frames, the type of information available consists mainly of motion vector information. For this reason, the tracking procedure differs according to the frame type.

The proposed face tracking algorithm is illustrated in Figure 1; in the figure, the location of one face (represented with a dark solid rectangle) is determined over a sequence of frames in a compressed video sequence. In I-frames, where DCT information is available, the presence and location of faces is determined based on the frame's colour and luminosity (as provided by the DC coefficients) and frequency (as provided by AC coefficients) properties. In all remaining frames, all detected faces are tracked based on the properties of existing motion vectors.

Since in the P- and B-frames available information typically consists of motion vector information, motion-vectorbased object tracking is performed to determine the location of previously detected faces.

\subsection{Face detection in the compressed domain}

The compressed domain face detection algorithm proposed in this paper uses a feature analysis-based approach to determine the presence and location of multiple frontal and rotated faces. This procedure is based purely on compressed domain information available in I-frames of MPEG-1 or

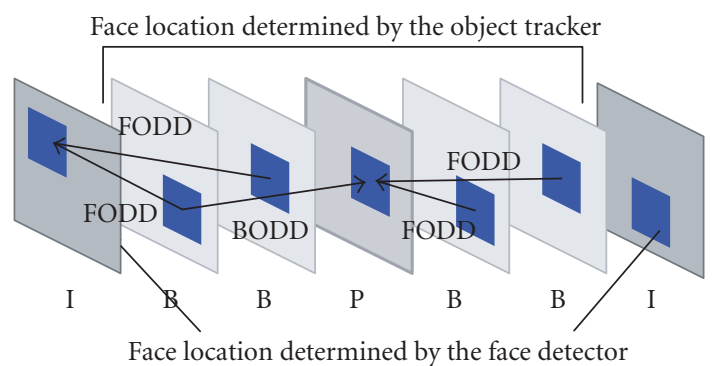

FIgure 1: The face tracking algorithm: the object's location in Pand $\mathrm{B}$-frames is determined using FODD and BODD techniques.

MPEG-2 video sequences (or in JPEG still images). The underlying assumption behind the feature analysis-based approach is that properties or features exist that are invariant from face to face. Thus, by detecting specific features such as skin colour and facial features, the presence and location of faces can be determined.

The diagram in Figure 2 represents an overview of the face detector's architecture and each of the algorithm's steps. In the figure, an example of the input and output of each step is illustrated.

The face detector's input corresponds to some AC coefficients and to all DC coefficients taken from the compressed image where detection is to be performed. In order to make the face detection algorithm as robust and independent as possible of the image or video capturing conditions, automatic contrast adjustment is applied to each input DC image. Afterwards, skin colour segmentation is applied to the DC colour image resulting from the contrast adjustment stage by determining the Mahalanobis distance $[10,11]$ between each pixel's value in the DC image and a skin colour model. This skin colour model is built beforehand from the statistical properties, in the normalized RGB colour space, of a large set of manually segmented faces. A binary closing operation with a $3 \times 3$ square structuring element, followed by a hole filling operation are applied on the binary image that resulted from the segmentation stage; these binary morphological operations are applied to ensure that in most cases a face is completely covered by a binary mask, without any holes in it. An algorithm to label binary connected regions is then applied in order to identify connected regions in the image.

It is known that among all facial features, the eyes/eyebrows and the mouth are the most prominent for face detection, recognition, and pose estimation [11-13]. Therefore, a series of image processing operations are applied in order to enhance the location of these facial features in the image such that existing faces may be located. The facial features' brightness properties are explored by computing grey scale dilated and eroded images from the contrast adjusted luminosity component of the input image. On the other hand, the facial features' variance properties are explored using frequency information provided by some AC coefficients. Taking into account the knowledge that specific sets of DCT-AC coefficients represent certain directional variations in the images [14], AC energy maps are built to emphasize the location 


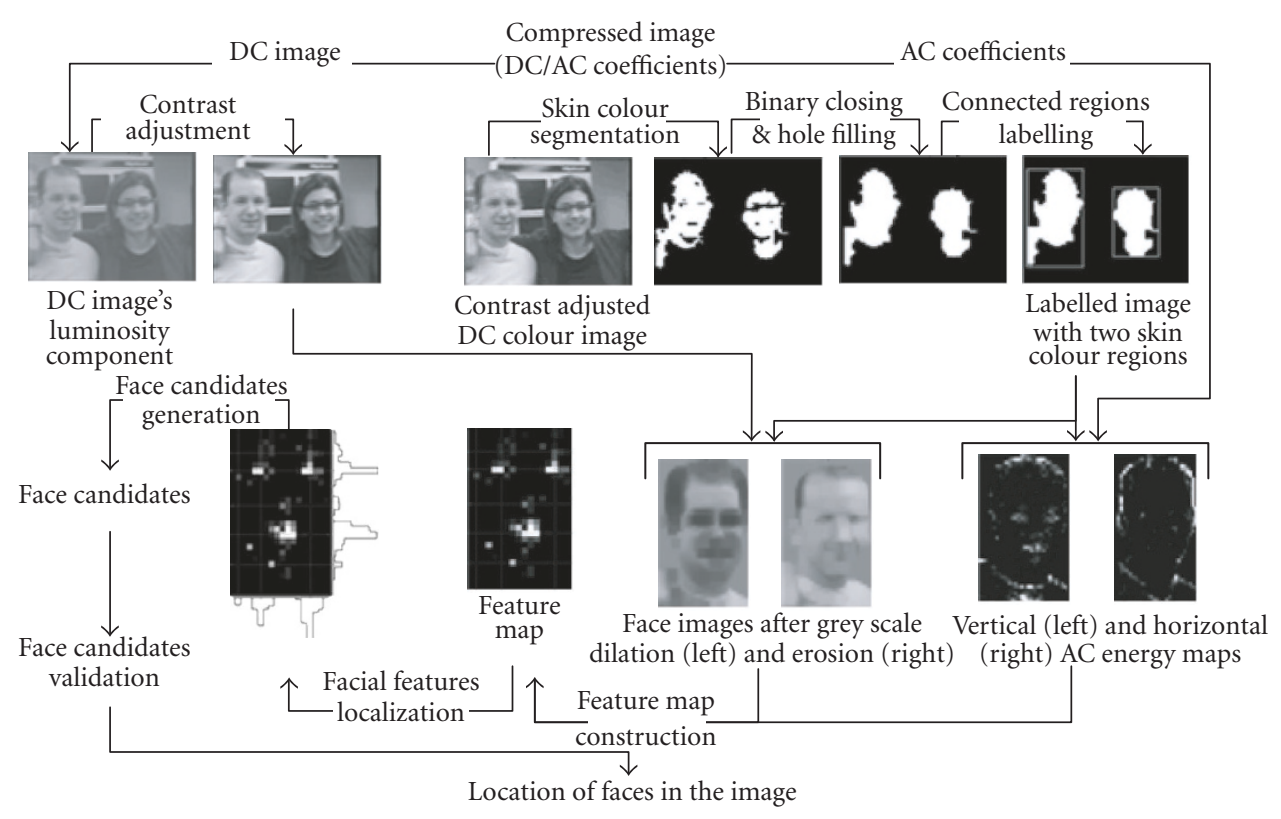

FIGURE 2: Face detector algorithm's architecture and examples of the input and output of each of the algorithm's steps.

of specific facial features. The vertical AC energy map, illustrated in Figure 2, is built from the AC coefficients indicated in Figure 3(a) and highlights regions of the image that have high vertical variance; on the other hand, the horizontal AC energy map also illustrated in Figure 2 is built from the coefficients indicated in Figure 3(b) and highlights regions with high horizontal and diagonal variance.

Finally, a feature map is built calculating for each position in the feature map matrix the following value:

$$
\begin{aligned}
\text { feature } \operatorname{map}(x, y)= & \frac{\operatorname{dilated}(x, y)}{\operatorname{eroded}(x, y)+1} \\
& \times \frac{\operatorname{vertical~energy~} \operatorname{map}(x, y)+1}{\operatorname{horizontal} \text { energy } \operatorname{map}(x, y)+1} .
\end{aligned}
$$

The first fraction in (1) enhances dark locations surrounded by bright areas (a similar equation is used in [11] to determine the location of the eyes in uncompressed images). The second fraction enhances regions with a high vertical variance and with a low horizontal variance thus highlighting the presence of facial features (e.g., eyes, eyebrows, mouth) and de-emphasizing locations like the sides of the face (which may as well have high horizontal variance).

In order to determine the location of faces that may exist in each skin colour region previously identified, the location of facial features is first determined. Facial features are identified directly from the feature map simply by projecting its values on vertical and horizontal axis (a similar technique was used in [12]). Face candidates representing possible locations of faces in the image can now be determined for each skin colour region. These candidates are generated from the location of the features determined from the feature map, according to a model of typical frontal and rotated human faces. Finally, face candidates are ranked after computing a relevance value to determine which best represents a face. Relevance determination will be based on the size of the face candidate, the face candidate's percentage of skin colour pixels and the face candidate's facial features intensity in the feature map. After computing the relevance of all generated face candidates for each individual skin colour region, the best is determined by choosing the one with the highest relevance for each individual skin colour region.

The face detection algorithm presented here is described in higher detail in $[15,16]$.

\subsection{Object tracking}

As explained before, object tracking is used in the face tracking system to determine, in $\mathrm{P}$ - and $\mathrm{B}$-frames, the location of previously detected faces. This tracking procedure is performed using only compressed domain information-in this case, motion vector information available in macroblocks in these frames.

However, a different type of motion vector information may be available according to the type of frames the objects are being tracked to. In fact, in P-frames only forward motion vector information may be available (indicating the prediction of the frame's macroblocks in past reference frames); in B-frames, both forward and backward (indicating the prediction of the frame's macroblocks in future reference frames) motion vector information may be available. For this reason, the tracking procedure varies, according to the type of motion vector information being used: when using backward motion vector information, objects are tracked using a backward object-displacement determination technique (as represented by BODD in Figure 1) - backward motion vectors are used to determine the object's displacement from a B-frame to a future reference P-frame; when using forward 


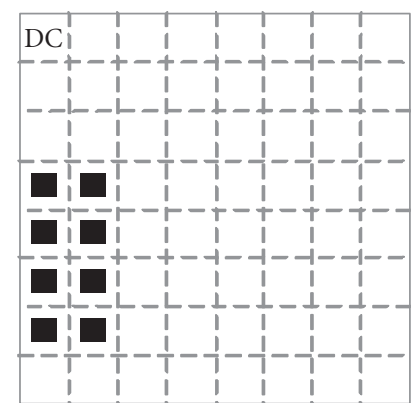

(a)

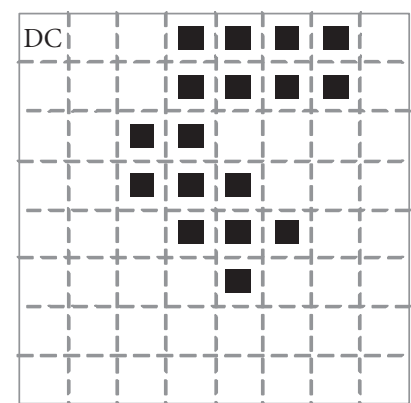

(b)

FIGURE 3: Set of AC coefficients for (a) vertical and (b) horizontal and diagonal variance.

motion vector information, objects are tracked using a forward object displacement determination technique (as represented by FODD in Figure 1) - forward motion vectors are used to determine the object's displacement from a previous reference (I- or P-) frame to a B-or P-frame. Both techniques are described in the following.

\subsubsection{Backward object displacement determination}

Defining SMB as the set of macroblocks (MBs) that comprise the object let $S M V$ represent the set of backward motion vectors (BMVs) corresponding to the macroblocks that define the object,

$$
S M V=\left\{M V_{1}, \ldots, M V_{n}\right\},
$$

where $n$ is the number of macroblocks that define the object being tracked. If this set is empty $(n=0)$, meaning that no BMVs exist for the MBs that comprise the object, no object displacement vector can be determined and the face cannot be tracked further.

Let a motion vector be defined by its $x$ - and $y$-components, that is,

$$
M V=(M V x, M V y) .
$$

The sets $S M V x$ and $S M V y$ can now be defined, representing the set of $x$ - and $y$-components, respectively, for the motion vectors corresponding to the macroblocks that comprise the object being tracked:

$$
\begin{aligned}
& S M V x=\left\{M V x_{1}, \ldots, M V x_{n}\right\}, \\
& S M V y=\left\{M V y_{1}, \ldots, M V y_{n}\right\} .
\end{aligned}
$$

In order to determine the object's displacement, a displacement vector is determined from the mean information associated with the set of the object's backward motion vectors.

The mean backward displacement vector, $D V_{\text {mean }}$, is a vector whose $x$ - and $y$-components are determined as the average $^{1}$ of the $x$ - and $y$-components of the motion vectors taken from the $S M V x$ and $S M V y$ defined before, that is,

$$
D V_{\text {mean }}=(\overline{D V x}, \overline{D V y})
$$

with

$$
\overline{D V x}=\frac{\sum_{i=1}^{n} M V x_{i}}{n}, \quad \overline{D V y}=\frac{\sum_{i=1}^{n} M V y_{i}}{n},
$$

where $M V x_{i}$ and $M V y_{i}$ are the $i$ th values of the $S M V x$ and $S M V y$ sets defined before.

If the objects are not moving, the mean backward displacement vector will have both components equal to zero and the tracking algorithm will simply indicate the same location as indicated in the previous frame - the algorithm should not fail to track faces when they are not moving.

Although the proposed algorithm is not used to track objects to I-frames (since object-in this case, face-detection takes place in those frames), it should be noted that the backward object displacement determination technique just described could be used to determine the object's displacement from a B-frame to a future reference I-frame-in case no detection algorithms were available, the proposed solution would be much less computationally complex than the technique proposed in [6] where block matching is needed in order to extract motion features for the I-frames.

Additionally, it should be noted that the computation of displacement vectors from backward motion vectors actually increases the tracking algorithm's robustness to strong motion: by allowing for an object's position in a P-frame to be determined from a B-frame rather than from a previous $\mathrm{P}$ or I-frame, the number of frames between the frame where the object is being tracked from to the frame where the object is being tracked to can be much smaller. This enhancement will be particularly noticeable in sequences where between

\footnotetext{
${ }^{1}$ Besides the average, the mode and the median information were also computed. Experiments have shown, however, that using the average will yield the best results.
} 
each reference (I- or P-) frame, a relatively high number of B-frames exist (e.g., I B B B P B B B ...)-in this case, the Pframe is distant from the previous reference frame and thus, the motion vectors may not be able to correctly express an object's motion, especially if the motion is strong.

\subsubsection{Forward object displacement determination}

For each macroblock in a P- or B-frame, a forward motion vector may exist to indicate the prediction of the location of that macroblock in a previous I- or P-frame. In the proposed object tracking algorithm, forward motion vectors are used to track objects to B-frames; additionally, they can be used to track objects to P-frames in case backward objectdisplacement determination techniques are not being used or in case no backward motion vectors exist that allow the object to be tracked from a B-frame to a P-frame. In any case, forward motion vectors allow for an object's displacement from an I- or P-frame to a B- or P-frame to be determined.

The object may be defined as a set of macroblocks in previous reference I- or P-frames. However, (forward) motion vector information is only available for macroblocks in the current frame. On the other hand, the object can only be tracked from the previous reference frame to current frame if the macroblocks - which comprise it-have some kind of motion information that predicts in some way their location in the current frame. Because the motion information is not readily available, the problem of determining a displacement vector using forward motion information is much different from that found while determining a displacement vector using backward motion vector information (as described in the previous section).

The displacement vector will also be determined from a set of motion vectors. However, since motion vector information-relating the frame where the object is being tracked from to the frame where the object is being tracked to-is available only in the latter, forward motion information needs to be somehow "projected" to the reference frame. In other words, each macroblock that defines the object in the reference frame will need a kind of motion vector that indicates its "backward" prediction in the current frame. For that purpose, a forward motion vector map is built for the reference frame, based on which the forward object's displacement vector will be determined. This forward motion vector map consists of a set of lists, built for each macroblock in the reference frame. Each one of these lists corresponds to a set of inverted forward motion vectors (IFMVs) representing the backward prediction of a reference frame's macroblock in the current frame and an associated "confidence" value for that prediction.

Following the nomenclature used in Figure 4, the forward motion vector map determination procedure will now be explained.

A macroblock $i$ is defined by the $x$ - and $y$-coordinates of its leftmost and topmost pixel, $M B x_{i}$ and $M B y_{i}$, respectively

$$
M B_{i}=\left(M B x_{i}, M B y_{i}\right)
$$

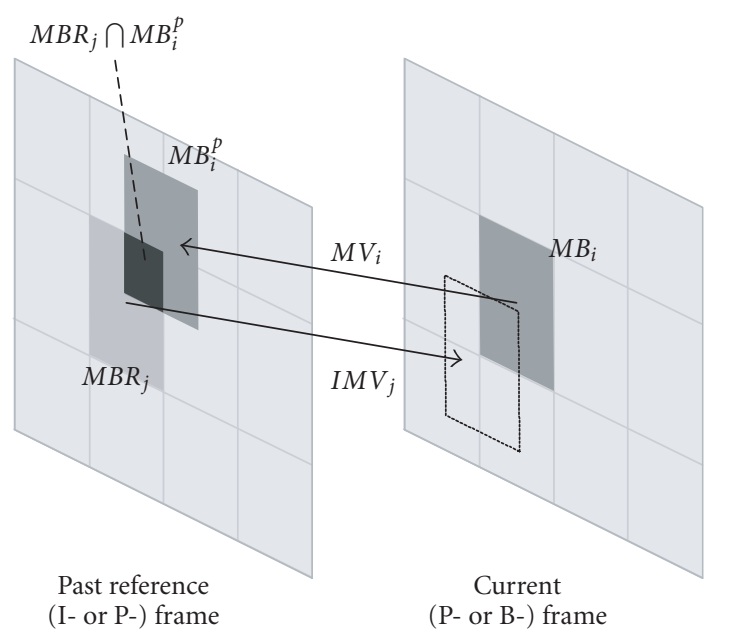

FIGURE 4: Forward motion vector map determination.

and by its dimensions which are constant and correspond to 16 luminance pixels of both width and height. ${ }^{2}$

Let $M V_{i}$ represent the forward motion vector of macroblock $i$ in the current (P- or B-) frame,

$$
M V_{i}=\left(M V x_{i}, M V y_{i}\right),
$$

where $M V x_{i}$ and $M V y_{i}$ are its $x$ - and $y$-components, respectively.

Using the forward motion vector $M V_{i}$ from the current frame, macroblock $i$ can be "projected" to the reference frame simply by shifting that macroblock's position by the corresponding forward motion vector. Let $M B_{i}{ }^{p}$ represent the projection of macroblock $i$ in the reference frame,

$$
M B_{i}^{p}=\left(M B x_{i}+M V x_{i}, M B y_{i}+M V y_{i}\right),
$$

and let $M B R_{j}$ represent a macroblock $j$ in the reference frame that is overlapped by the projection of macroblock $i^{3}$ in the reference frame, such that

$$
M B R_{j} \cap M B_{i}^{p} \neq \phi
$$

with $M B R_{j}$ defined by the coordinates of its leftmost topmost pixel,

$$
M B R_{j}=\left(M B R x_{j}, M B R y_{j}\right) .
$$

Now, let $I M V_{j}$ represent the inverted forward motion vector of a current frame's macroblock $i$ overlapping macroblock $M B R_{j}$ in the reference frame

$$
I M V_{j}=\left(I M V x_{j}, I M V y_{j}, O P_{j}\right)
$$

\footnotetext{
2 The macroblocks' dimensions for the chrominance components can be different: if subsampled horizontally by 2 , each macroblock's width will correspond to 8 pixels; if subsampled vertically by 2 , each macroblock's height will correspond to 8 pixels.

${ }^{3}$ A minimum of one and a maximum of four macroblocks in the reference frame may be overlapped by the projection of a macroblock from the current frame to the reference frame.
} 


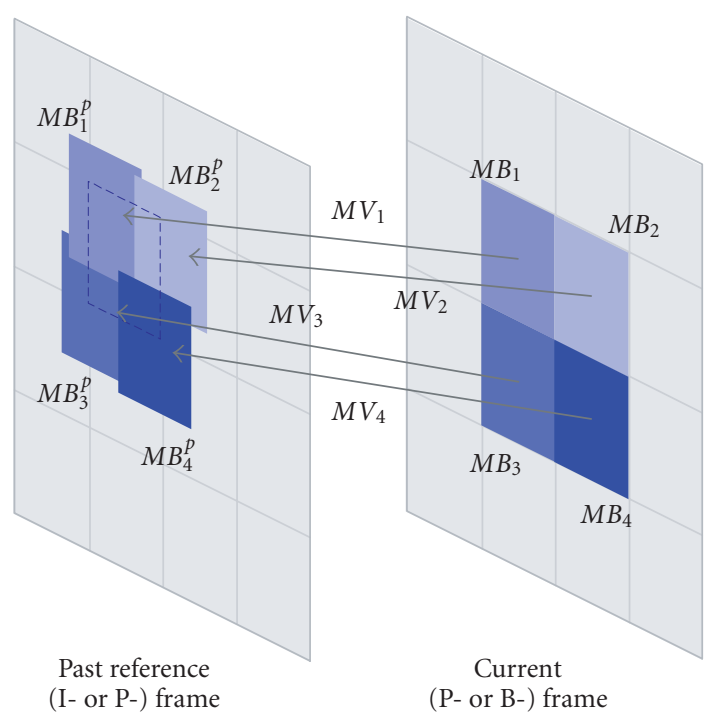

(a)

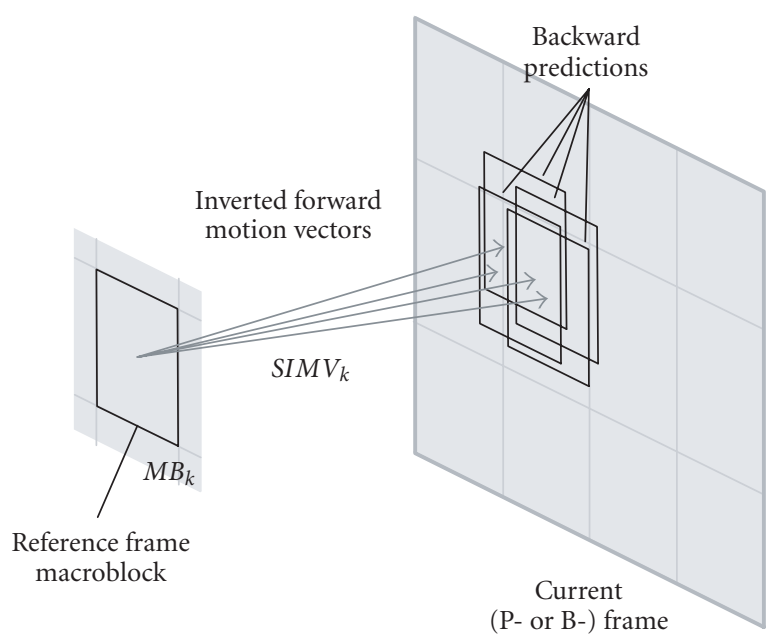

(b)

Figure 5: (a) Projection of four current frame's macroblocks on a past reference frame; (b) inverted forward motion vectors determined for the reference frame macroblock indicated in (a) and its corresponding backward predictions on the current frame.

with

$$
\begin{gathered}
I M V x_{j}=-M V x_{i}, \quad I M V y_{j}=-M V y_{i}, \\
O P_{j}=\operatorname{area}\left(M B R_{j} \cap M B_{i}^{p}\right),
\end{gathered}
$$

where $I M V x_{j}$ and $I M V y_{j}$ represent the $x$ - and $y$-components of the inverted forward motion vector from macroblock $i$ and $O P_{j}$ represents the overlap percentage value indicating "how much" of the projected macroblock $M B_{i}{ }^{p}$ overlaps $M B R_{j}$.

Figure 5(a) illustrates the projection of four macroblocks from the current frame to a past reference frame; associated with each of these macroblocks is a forward motion vectoras can be seen, the macroblock in the reference frame indicated with the black dotted square is overlapped by four projections. Figure 5(b) illustrates the list of inverted forward motion vectors determined for that macroblock in the reference frame-since more than one projection overlapped the macroblock (as in Figure 5(a)), more than one inverted forward motion vector exists for that reference frame macroblock; these inverted forward motion vectors thus indicate an estimate of the (backward) prediction of the reference frame macroblock in the current frame.

A complete forward motion vector map will consist of a list of inverted forward motion vectors, like those defined in (12), for each macroblock in the reference frame-the list of inverted forward motion vectors for the macroblock $M B_{k}$ in Figure 5(b) corresponds to the set of inverted forward motion vectors and the associated overlap percentages, indicated as $S I M V_{k}$.

For each of these macroblocks, the list of inverted forward motion vectors may have more than one element since more than one macroblock in the current frame could have had a projection overlapping the same macroblock in the reference frame. Like described before, associated with each inverted forward motion vector, is an overlap percentage value indicating "how much" of the macroblock was overlapped by the current frame projected macroblock to which the inverted motion vector corresponded. It follows naturally that the higher the overlap percentage value is for an inverted motion vector, the more likely it will be that the vector indicates a good backward prediction for that macroblock in the current frame. On the contrary, a low overlap percentage value associated with an inverted forward motion vector will give no guarantee whatsoever about the "quality" of the backward prediction for that macroblock in the current frame. This overlap percentage value thus indicates a "confidence" value regarding the backward prediction of a given macroblock in the reference frame.

The displacement of an object from the reference frame to the current frame can now be determined from the set of inverted forward motion vectors. Defining the object as a set of macroblocks in the reference frame, the set of inverted forward motion vectors from where the object's displacement is computed corresponds simply to the union of the sets of inverted forward motion vectors, SIMV $V_{k}$, for each of the macroblocks that comprise the object.

Let $S M B$ represent the macroblocks that define the object in the reference frame. Now, let $S I M V_{k}$ represent the set of inverted forward motion vectors for macroblock $k$ in the reference frame,

$$
S I M V_{k}=\left\{I M V_{k 1}, \ldots, I M V_{k \text { kmax }}\right\},
$$

where $I M V_{k}$ was defined before, in (12) and kmax corresponds to the number of inverted forward motion vectors that macroblock $k$ has in its list in the forward motion vector map. 
TABLE 1: Face detection and face tracking algorithms' complexity.

\begin{tabular}{lcccc}
\hline & \multicolumn{2}{c}{ Face detection } & \multicolumn{2}{c}{ Face tracking } \\
\hline Resolution & MIPS & Mcycle/s & MIPS & Mcycle/s \\
\hline PAL D1 & 7.53 & 20.95 & 9.22 & 25.95 \\
VGA & 5.58 & 15.52 & 6.83 & 19.22 \\
SIF & 1.53 & 4.27 & 1.88 & 5.29 \\
\hline
\end{tabular}

A global set of inverted motion vectors for the entire object, SIMV, can now be built from the union of the sets $S I M V_{k}$ for the various macroblocks that define the object in the reference frame,

$$
S I M V=\bigcup_{m} S I M V_{m} \quad \forall_{m}: M B_{m} \in S M B
$$

Situations may occur where no inverted forward motion vectors exist in the global set SIMV. This situation occurs if the macroblocks in the current frame have no forward motion vectors. In this case, no forward object displacement can be determined, the object's location in the current frame cannot be determined and naturally faces cannot be tracked further.

In order to determine the object's displacement, a displacement vector is determined from the mean ${ }^{4}$ information of the global set of inverted forward motion vectors, SIMV. The mean inverted forward displacement vector, $I D V_{\text {mean }}$, is a vector whose $x$ - and $y$-components are determined as the average of the $x$ and $y$ component values from the global set SIMV defined before, weighted by the corresponding overlap percentage values, that is,

$$
I D V_{\text {mean }}=(\overline{I D V x}, \overline{I D V y})
$$

with

$$
\begin{aligned}
& \overline{I D V x}=\frac{\sum_{i=1}^{\mathrm{qmax}} I M V x_{q} \cdot O P_{q}}{\sum_{q=1}^{\mathrm{qmax}} O P_{q}}, \\
& \overline{I D V y}=\frac{\sum_{i=1}^{\mathrm{qmax}} I M V y_{q} \cdot O P_{q}}{\sum_{q=1}^{\mathrm{qmax}} O P_{q}},
\end{aligned}
$$

where qmax is the number of inverted motion vectors in the global set SIMV, and IMV $x_{q}$ and $I M V y_{q}$ represent the $x$ and $y$-components, respectively, of the $q$ th inverted forward motion vector in SIMV.OP represents the overlap percentage value associated with the $q$ th inverted forward motion vector in SIMV.

As explained, the overlap percentage value provides an indication of the "confidence" associated with the prediction of a reference frame's macroblock in the current frame. Therefore, by weighting the average with this overlap percentage value, inverted forward motion vectors with higher

\footnotetext{
${ }^{4}$ Besides the mean, the mode and the median information were also computed from the set of inverted forward motion vectors. Experiments have shown, however, that using the mean computation will yield the best results.
}

"confidence" will weigh more in the computation of the object's displacement vector whereas lower "confidence" motion vectors will influence the displacement vector's computation less.

Again, if the objects are not moving, the mean inverted forward displacement vector will have both components equal to zero and the tracking algorithm will simply indicate the same location as indicated in the previous frame-the algorithm should not fail to track faces when they have stopped moving.

\section{COMPUTATIONAL COMPLEXITY AND PERFORMANCE ANALYSIS}

In order to determine the face tracking algorithm's computational complexity, that is, the required number of instructions per second, face tracking (comprising both the face detector and the object tracker) is considered to be applied on an ARM9-based platform, performing real-time tracking across all frames of an MPEG-2 video sequence, with a frame rate of 25 frames per second and a GOP size of 6 . The face detection (alone) and face tracking algorithms' computational complexities - measured in millions of instructions per second (MIPS) and in millions of cycles per second (Mcycle/s) - are indicated in Table 1. As it can be easily seen, the face detection algorithm alone is extremely inexpensive, being able to detect and locate faces in I-frames of compressed video sequences. With a small increase (approximately $25 \%$ for PAL D1 resolution) of complexity, the complete face tracking algorithm (face detection and object tracking) is able to determine the location of faces in all frames of compressed video sequences. The proposed algorithms are thus extremely inexpensive and adequate for use in low-performance CE devices, using less than $10 \%$ of an ARM9 processor's capacity for face tracking in frames with VGA resolution.

In order to analyse the algorithm's tracking performance, tests were performed on two home-video sequences-indoors and outdoors. The sequences were captured under difficult and heterogeneous light conditions. The subjects were recorded with frontal and nonfrontal poses and with different facial expressions, a variety of hairstyles, and some of the subjects had a beard and glasses. Both sequences were encoded in MPEG-2 with a GOP size of 6 . A face is considered to be detectable if it is tilted below an angle of \pm 20 degrees, rotated below an angle of \pm 80 degrees and if most of the face's colour corresponds to skin colour.

In order to evaluate the face tracking algorithm's performance, a loose location criterion is introduced. According to 
TABLE 2: Face detection and face tracking performance results.

\begin{tabular}{|c|c|c|c|c|c|c|c|c|c|c|c|c|c|c|}
\hline \multirow[b]{2}{*}{$\begin{array}{c}\text { Test } \\
\text { sequence }\end{array}$} & \multicolumn{7}{|c|}{ Face detection } & \multicolumn{7}{|c|}{ Face tracking } \\
\hline & Frames & Faces & $\begin{array}{l}\text { Correct } \\
\text { locations }\end{array}$ & $\begin{array}{c}\text { Missed } \\
\text { faces }\end{array}$ & $\begin{array}{c}\text { False } \\
\text { locations }\end{array}$ & Recall & Precision & Frames & Faces & $\begin{array}{l}\text { Correct } \\
\text { locations }\end{array}$ & $\begin{array}{c}\text { Missed } \\
\text { faces }\end{array}$ & $\begin{array}{c}\text { False } \\
\text { locations }\end{array}$ & Recall & Precision \\
\hline Indoors & 736 & 335 & 311 & 24 & 68 & 0.93 & 0.82 & 4416 & 1973 & 1771 & 202 & 502 & 0.90 & 0.78 \\
\hline Outdoors & 541 & 159 & 149 & 10 & 34 & 0.94 & 0.81 & 3246 & 950 & 868 & 82 & 230 & 0.91 & 0.79 \\
\hline Global & 1277 & 494 & 460 & 34 & 102 & 0.93 & 0.82 & 7662 & 2923 & 2639 & 284 & 732 & 0.90 & 0.78 \\
\hline
\end{tabular}
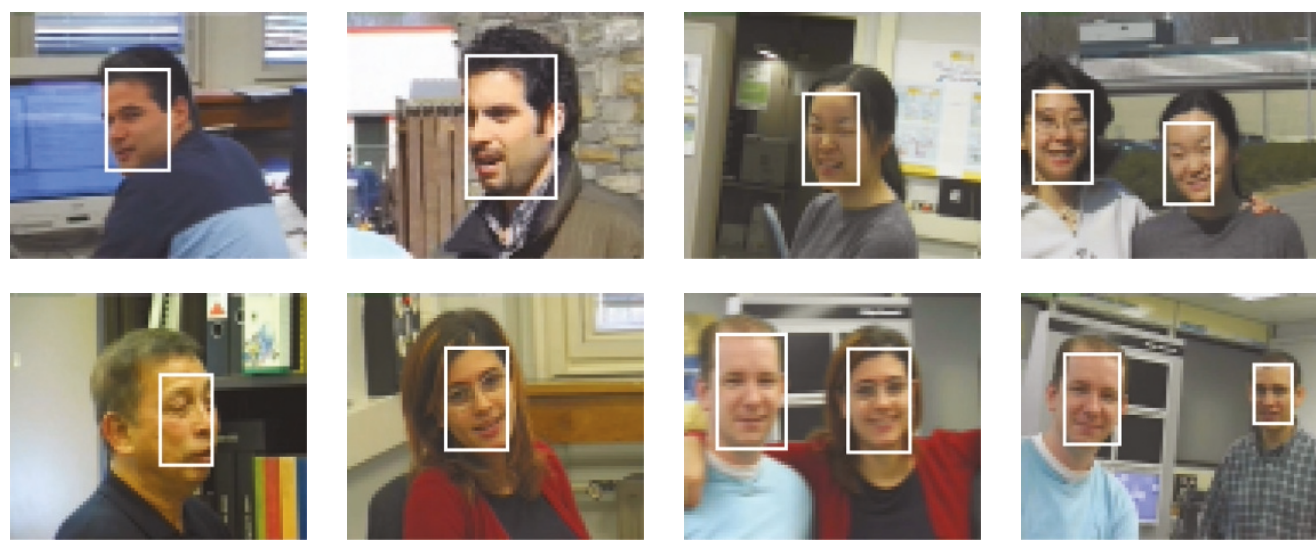

FIgURE 6: Examples of faces correctly detected with the described algorithm.

this criterion, the location of faces in each frame are manually annotated and classified according to the following classes:

(i) a correct location is considered when the bounding box representing the face location as determined by the face tracking algorithm covers approximately at least half of the face or encloses completely at least one visible facial feature;

(ii) a false location is considered when the bounding box representing the face location does not satisfy any of the two previous conditions, that is, when something that is not a face is erroneously located as such;

(iii) a missed face is considered when a face appears in the image, it is considered to be detectable but it is not under the bounding box of a correct location.

The tracking algorithm's performance, according to this matching criterion, will be evaluated in terms of the ratio of correct locations against the number of detectable faces, as expressed by the recall metric,

$$
\text { recall }=\frac{\text { correct }}{\text { correct }+ \text { missed }},
$$

where correct and missed correspond to the number of correct and missed locations, respectively, classified according to the criterion defined above.

Additionally, the algorithm's performance will also be evaluated in terms of the ratio between the number of correct detections and the total number of detections (i.e., correct and false detections), as expressed by the precision metric,

$$
\text { precision }=\frac{\text { correct }}{\text { correct }+ \text { false }} \text {, }
$$

where correct and false correspond to the number of correct and false locations, respectively, classified according to the criterion defined above. The precision metric expresses the capability of the tracking algorithm to determine only correct locations while avoiding false alarms. Notice that the recall metric expresses the capability to locate all the detectable faces regardless of the number of false locations indicated.

The performance results for both the face detection and the object tracking algorithms were obtained after visually analysing the detection and tracking results on each single frame and, using the criterion defined above, counting the number of correct and false locations and the number of missed faces.

Table 2 indicates the detection (in I-frames) and the tracking results (in all frames) for each of the two video sequences. As it can be seen, the face detection algorithm achieves a very high recall value of $93 \%$ and a slightly lower precision value of $82 \%$. The performance of the tracking algorithm naturally depends on the performance of the detection algorithm (faces that are not detected will not be tracked and false detections will naturally lead to false locations by the tracking algorithm). By using only motion information to track faces throughout each GOP, the tracking algorithm achieves a still extremely high recall value of $90 \%$ and a precision value of $78 \%$. The performance decreases only slightly when compared to the detection performance. However, the location of faces may now be determined for all frames of the video sequences.

Figure 6 illustrates examples of correctly detected faces with the described face detection algorithm. As it can be seen, 


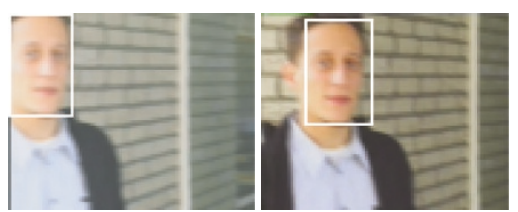

(a)

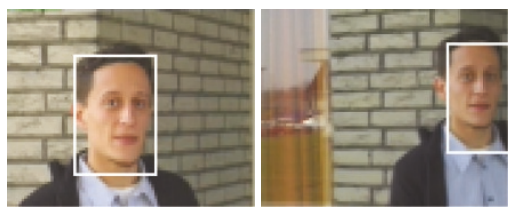

(d)

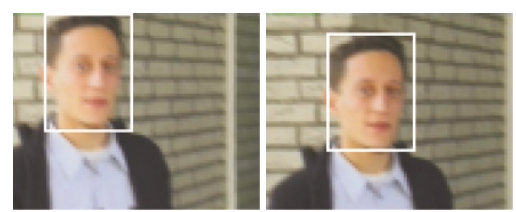

(b)

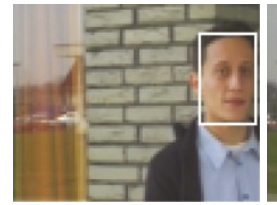

(e)

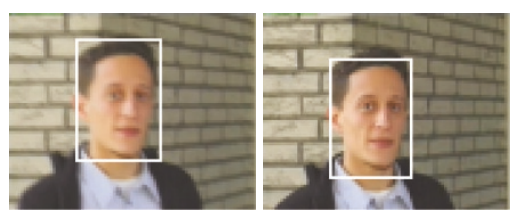

(c)
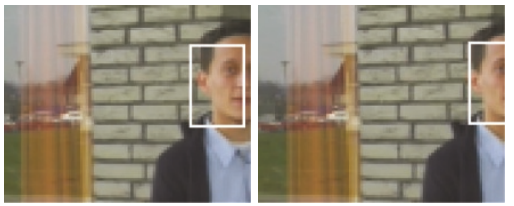

(f)

FIGURE 7: Example of a face correctly tracked for the entirety of a shot comprised of 22 GOPs; for each of the indicated GOPs, two figures are illustrated: the result of detection in the first (I) frame of the GOP (on the left) and the result of tracking at the last frame of the GOP (on the right). (a) GOP1, (b)GOP2, (c) GOP3, (d) GOP20, (e) GOP21, and (f) GOP22.
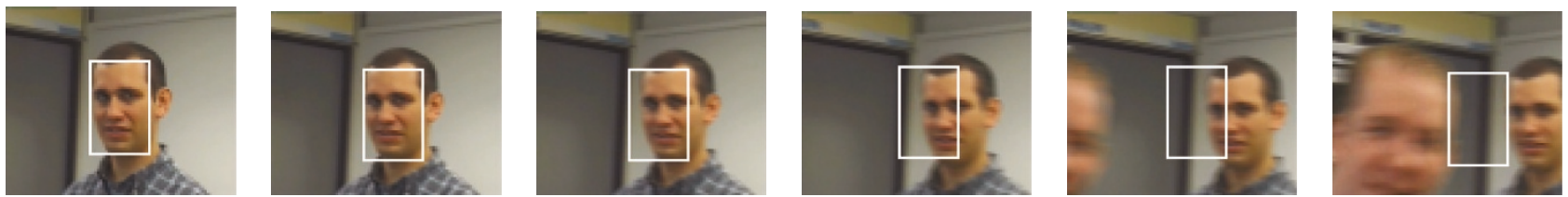

FIGURE 8: Example of the result of face tracking in a GOP with strong camera motion; the bounding box in the first frame, in the left, corresponds to the result of face detection.

the detection algorithm is able to detect rotated and titled faces.

Figure 7 illustrates an example of a correctly tracked face for an entire shot comprised of 22 GOPs. The tracking algorithm is able to cope with camera pan, zoom, and even partial occlusion of the face. Figure 8 illustrates an example of a sequence where the face tracking algorithm is not able to correctly locate the face being tracked throughout all frames of a GOP with strong camera motion. This is due to the fact that in this particular sequence the motion vectors are not able to correctly express the objects' motion, and thus, tracking is not performed correctly. The same figure highlights another situation where the tracking algorithm naturally fails, that is, when a new face appears in the middle of the GOP. Naturally, since the only faces that can be tracked are those that were detected, a newly appearing face will only be tracked from the moment it is detected in an I-frame.

\section{CONCLUSIONS}

This paper proposed a compressed domain object tracking algorithm, which, combined with a face detection algorithm that also acts in the compressed domain, allows for faces to be tracked throughout video sequences where a specific type of compressed domain data is available (such as MPEG-1 or MPEG-2 video sequences). Using only DCT coefficients to detect faces in some frames and motion information as provided by forward and backward motion vectors in the remaining frames, the proposed algorithm offers a processing power efficient, inexpensive, fast, and sufficiently reliable solution for object tracking for various consumer electronic applications. The solution's processing power efficient nature enables even its implementation on portable and mobile platforms for applications such as face image stabilization for mobile video conferencing.

The complete face tracking algorithm's performance was analysed after performing face tracking on two home-video sequences. These sequences were considered to be representative of the typical content in applications for which the developed face tracking algorithm was originally devisedcompressed home-video sequence analysis for content management in low-power CE devices. The face tracking algorithm's recall measure was found to be high $(90 \%)$ while its precision was found to be slightly lower $(78 \%)$. While it was shown that the tracking algorithm depends on the performance of the detection algorithm, its performance was found not to decrease significantly when compared to the latter. In fact, with only a small increase of complexity, the location of faces may be determined with sufficient accuracy for all frames in compressed video sequences.

A comparison with other algorithms proposed in the literature is hard since few face tracking algorithms exist that perform in the compressed domain. In addition, among those described, performance is not tested in common and 
publicly available content nor is there any publicly available code of their implementations. For future work, the proposed algorithm's performance will be analysed under known video sequences and possible comparisons will be made against existing face detection and face tracking algorithms.

In conclusion, the novelty of the face tracking algorithm proposed resides in the use of backward motion vectors to allow tracking to be performed from B-frames to (I- or P-) reference frames. The algorithm should thus be more robust to strong motion than previously proposed algorithms. Besides, it now allows for the tracking of objects to I-frames.

Additionally, the algorithm cleverly uses inverted motion vectors weighted by overlap areas to track objects using forward motion vectors. This strategy allows for the computation of a weighted average of inverted motion vectors, where the weight is given by the overlap percentage value, which, as explained, acts as an indication of "confidence." Thus, motion vectors with higher confidences will weigh more in the displacement vector computation. This technique has been experimentally proven to yield better performance than the typical mode value computation used in previously proposed algorithms. Besides being sounder, it is much less computationally complex than existing techniques.

\section{REFERENCES}

[1] E. Hjelmås and B. K. Low, "Face detection: a survey," Computer Vision and Image Understanding, vol. 83, no. 3, pp. 236-274, 2001.

[2] M.-H. Yang, D. J. Kriegman, and N. Ahuja, "Detecting faces in images: a survey," IEEE Transactions on Pattern Analysis and Machine Intelligence, vol. 24, no. 1, pp. 34-58, 2002.

[3] R. Achanta, M. Kankanhalli, and P. Mulhem, "Compressed domain object tracking for automatic indexing of objects in MPEG home video," in Proceedings of IEEE International Conference on Multimedia and Expo (ICME '02), vol. 2, pp. 61-64, Lausanne, Switzerland, August 2002.

[4] J. Wang, R. Achanta, M. Kankanhalli, and P. Mulhem, "A hierarchical framework for face tracking using state vector fusion for compressed video," in Proceedings of IEEE International Conference on Acoustics, Speech, and Signal Processing (ICASSP '03), vol. 3, pp. 209-212, Hong Kong, April 2003.

[5] D. Schonfeld and D. Lelescu, "VORTEX: video retrieval and tracking from compressed multimedia databases: affine transformation and occlusion invariant tracking from MPEG-2 video," in Storage and Retrieval for Image and Video Databases VII, vol. 3656 of Proceedings of SPIE, pp. 131-142, San Jose, Calif, USA, January 1998.

[6] L. Favalli, A. Mecocci, and F. Moschetti, "Object tracking for retrieval applications in MPEG-2," IEEE Transactions on Circuits and Systems for Video Technology, vol. 10, no. 3, pp. 427432, 2000.

[7] V. Mezaris, I. Kompatsiaris, N. Boulgouris, and M. G. Strintzis, "Real-time compressed-domain spatiotemporal segmentation and ontologies for video indexing and retrieval," IEEE Transactions on Circuits and Systems for Video Technology, vol. 14, no. 5, pp. 606-621, 2004.

[8] Y. Nakajima, A. Yoneyama, H. Yanagihara, and M. Sugano, "Moving object detection from MPEG coded data," in Visual Communications and Image Processing '98, vol. 3309 of
Proceedings of SPIE, pp. 988-996, San Jose, Calif, USA, January 1998.

[9] W.-N. Lie and R.-L. Chen, "Tracking moving objects in MPEG-compressed videos," in Proceedings of IEEE International Conference on Multimedia and Expo (ICME '01), pp. 965-968, Tokyo, Japan, August 2001.

[10] V. Vezhnevets, V. Sazonov, and A. Andreeva, "A survey on pixel-based skin color detection techniques," in Proceedings of International Conference on Computer Graphics \& Vision (GraphiCon '03), pp. 85-92, Moscow, Russia, September 2003.

[11] R.-L. Hsu, M. Abdel-Mottaleb, and A. K. Jain, "Face detection in color images," IEEE Transactions on Pattern Analysis and Machine Intelligence, vol. 24, no. 5, pp. 696-706, 2002.

[12] K. Sobottka and I. Pitas, "A novel method for automatic face segmentation, facial feature extraction and tracking," Signal Processing: Image Communication, vol. 12, no. 3, pp. 263-281, 1998.

[13] A. Nikolaidis and I. Pitas, "Facial feature extraction and determination of pose," in Proceedings of NOBLESSE Workshop on Non-Linear Model Based Image Analysis (NMBIA '98), pp. 257-262, Glasgow, Scotland, UK, July 1998.

[14] Y. Zhang and T.-S. Chua, "Detection of text captions in compressed domain video," in Proceedings of ACM Workshops on Multimedia (ACM MM '00), pp. 201-204, Los Angeles, Calif, USA, October 2000.

[15] P. Fonseca and J. Nesvadba, "Face detection in the compressed domain," in Proceedings of IEEE International Conference on Image Processing (ICIP '04), vol. 3, pp. 2015-2018, Singapore, October 2004.

[16] J. Nesvadba, R. Kleihorst, J. Fan, P. M. Fonseca, and H. Broers, "Face related features in consumer electronic (CE) device environments," in Proceedings of IEEE International Conference on Systems, Man and Cybernetics (SMC '04), vol. 1, pp. 641648, The Hague, The Netherlands, October 2004.

Pedro Miguel Fonseca has obtained his B.S. degree in electrical and computer engineering in 2002 from the Instituto Superior Técnico in Lisbon, Portugal. In 2004 he obtained his M.S. degree in electrical and computer engineering after completing an internship in Philips Research where he worked on face detection and face tracking in compressed video content. In 2004 he joined Philips Research as a Research Scien-

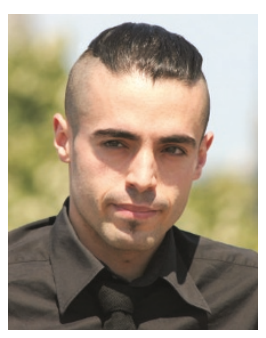
tist and has worked since then on algorithms for multimedia content analysis, pattern recognition, signal processing, and computer vision. He has also been involved in the design of system architectures for distributed content analysis and in the specification of future generations of ICs for video processing and encoding. He is actively involved in national and international research projects (MultimediaN, CANDELA) and has published several conference and journal papers in his field of research.

Jan Nesvadba obtained the Dipl.-Ing. (M.S.) degree (cum laude) in electrical engineering (telecommunication and information technology) from the Technical University of Vienna, Austria, in 1997. He did his M.S. thesis work in the field of electrobiology on dielectrophoresis of biological cells. Currently, he is finalizing his Ph.D. thesis at LaBRI, University of Bordeaux 1,

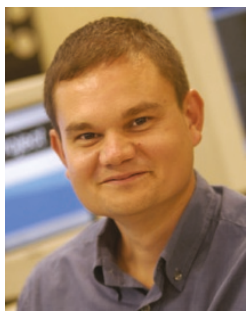


France. He joined Philips Research in 1998 and started his research career on digital return channels for HybridFiberCoax networks. His current fields of investigation as a Senior Scientist at Philips Research are retrieval algorithms (multimedia content analysis, computer vision, multimedia signal processing), related smart system architectures (e.g., distributed analysis for consumer electronics inhome networks, grid computing) for audiovisual content analysis, the adaptation of existing ICs (encoders, codecs) for the generation of content descriptors (e.g., MPEG-7), and the optimal use of these descriptors in consumer storage devices and user interfaces. He has published extensively in those fields (conferences, journal papers, book chapters, international patents), he is an active contribution member of various national and international projects (e.g., ITEA AVIR, ITEA CANDELA, Bsik MultimediaN), and he is an active committee member of multiple related conferences. 